\title{
Ileus Biliaire : A Propos D'un Cas Clinique
}

\author{
Saidou A, \\ Département de chirurgie, Hôpital National de Niamey Niger \\ Herrero A, \\ Département de Chirurgie Digestive et Transplantation Hépatique, \\ Hôpital Saint Eloi Montpellier \\ Ide $\mathrm{K}$, \\ James Didier L, \\ Younssa $\mathrm{H}$, \\ Sani R, \\ Département de chirurgie, Hôpital National de Niamey Niger
}

Doi: 10.19044/esj.2017.v13n30p529 URL:http://dx.doi.org/10.19044/esj.2017.v13n30p529

\begin{abstract}
Introduction: Gallstone ileus is a rare mechanical occlusion. It is caused by the enclosure of biliary macro lithiasis in a portion of the digestive tract resulting from a digestive bile fistula. We report a clinical case to discuss therapeutic modalities through a review of the literature. Medical observation: We report the case of a 77-year-old patient who was hospitalized in the Nephrology department of the university hospital in Montpellier for functional kidney failure and dehydration from vomiting and diarrhea. The none-injected abdominal-pelvic CT scan showed a gallstone ileus with 5 enclaved duodenum, jejunum and ileum lithiasis resulting into a small bowel obstruction. There are no signs of acute cholecystitis. The management was simple by enterolithotomy surgery alone after fixing of hydro electrolyte imbalance. The after surgery sequence was simple. Conclusion: Gallstone ileus is a rare surgical condition. The high mortality rate in the management of this condition makes enterolithotomy the least invasive and recommended method.
\end{abstract}

Keywords: Ileus-Biliary-Fistula

Résumé

Introduction: L'iléus biliaire est une occlusion mécanique rare. Elle est causée par l'enclavement de macro-lithiases biliaires dans une portion du tractus digestif issues d'une fistule bilio-digestive. Nous rapportons un cas clinique pour discuter des modalités thérapeutiques à travers une revue de la 
littérature. Observation : Nous rapportons le cas d'un patient âgé de 77 ans qui était hospitalisé dans le service de Néphrologie de CHRU de Montpellier pour une insuffisance rénale fonctionnelle et une déshydratation suite à des vomissements et une diarrhée. Le scanner abdomino-pelvien non injecté réalisé montrait un iléus biliaire avec 5 lithiases enclavées, duodénales, jéjunales et iléales donnant une occlusion haute. Il n'y a pas de signes de cholécystite aigue. La prise en charge a été simple par une enterolithotomie seule après la correction des troubles hydro électrolytiques. Les suites opératoires ont été simples. Conclusion : L'iléus biliaire est une affection chirurgicale rare. Le taux élevé de mortalité dans la prise en charge de cette pathologie fait que l'enterolithotomie reste la méthode la moins invasive et la plus recommandée.

Mots clés: Iléus-Biliaire-Fistule

\section{Introduction}

L'iléus biliaire est une pathologie ancienne, décrite depuis 1654 par ERASMUS, un physicien Danois sur une autopsie (Deskoff, 1955). Elle entraine une occlusion mécanique causée par l'obstruction de macro lithiase biliaire dans une portion du tractus digestif issue d'une fistule bilio digestive. C'est une pathologie rare et représente 1 à $4 \%$ de toutes les occlusions mécaniques (Halabi, 2014). Dans la littérature, la population féminine âgée est la plus concernée, avec un âge moyen de 65 ans (Halabi, 2014). Actuellement, le diagnostic est aisé avec le scanner abdominal et la prise en charge doit être urgente afin de limiter les complications et la mortalité qui sont très élevées. Plusieurs stratégies thérapeutiques ont été décrites dont l'enterolithitomie seule, le « one stage procédure », la résection intestinale et plus récemment la cœlioscopie assistée, le traitement endoscopique.

\section{Observation}

Nous rapportons le cas d'un patient âgé de 77 ans qui était hospitalisé dans le service de Néphrologie de CHRU de Montpellier pour une insuffisance rénale fonctionnelle et une déshydratation suite à des vomissements et une diarrhée. Au troisième jour d'hospitalisation, une radiographie pulmonaire avait été faite au lit et qui montrait une poche à air gastrique très dilatée, ceci motiva la réalisation d'un scanner abdomino pelvien non injecté qui avait montré une occlusion haute avec une distension $\mathrm{du}$ pylore et de l'estomac (figure $\mathrm{n}^{\circ} 1$ ) avec deux images hyperdenses circulaires intra luminales qui faisaient l'obstruction au niveau duodénal. On avait retrouvé ce même type d'images hyperdenses distales, deux au niveau jéjunal et une au niveau iléal. La plus grande faisait un diamètre de $5 \mathrm{~cm}$ 
(figure $\mathrm{n}^{\circ} 2$ ). Il n’y avait pas de signes de cholécystite aigue et la vésicule biliaire était alithiasique avec une aérobilie et une image de fistule vésico duodénale. Il n'y avait pas de distension intestinale mais une occlusion haute à partir du pylore. Au total, on avait retenu diagnostic de l'iléus biliaire sans occlusion franche avec un rectum aéré.

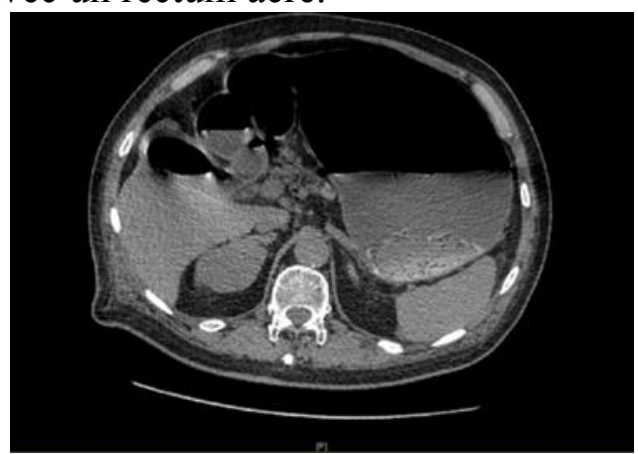

Figure n²: Scanner abdominal non injecté montrant une distension gastrique avec aérobilie (flèche bleue)

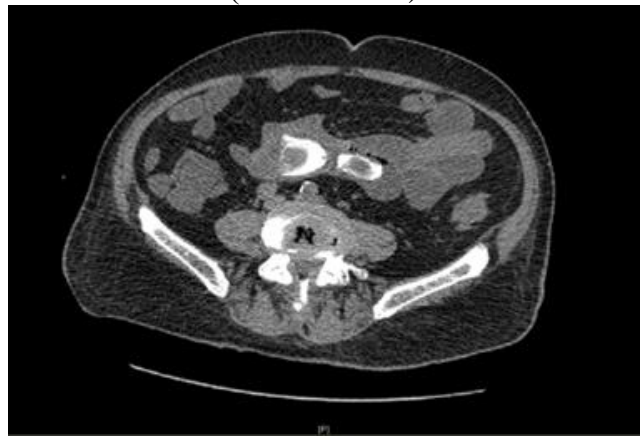

Figure n³: Scanner abdomino-pelvien non injecté montrant deux images hyperdenses calciques iléales (flèche rouge)

La réhydratation parentérale était poursuivie associée à une antibiothérapie sur des hémocultures positives. La fonction rénale s'était normalisée après 48 heures, mais il s'était installé un tableau occlusif avec arrêt des matières et des gaz. Une sonde naso gastrique avait été posée. Dans ces conditions, une indication chirurgicale a été posée. La voie d'abord était une cœlioscopie assistée première, puis devant l'échec, car les lithiases étaient très difficiles à mobiliser, une mini laparotomie médiane péri ombilicale a été réalisée (figure $\mathrm{n}^{\circ} 3$ ). Après avoir regroupé les 5 macrolithiases au niveau iléal, il a été réalisé une entérotomie longitudinale sur le bord anti mésentérique, ce qui a permis leur extraction (figure $\mathrm{n}^{\circ} 4$ et $\mathrm{n}^{\circ} 5$ ).

Suture iléale transversale avec des points séparés. L'étage sus mésocolique n'avait pas été manipulé, la vésicule biliaire et la fistule bilio duodénale n'avaient pas été explorées, au risque de créer d'autres lésions. 


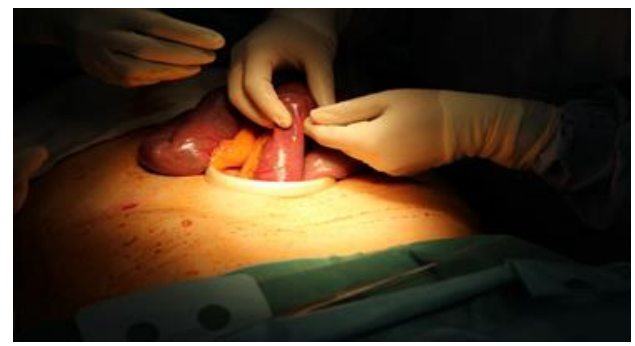

Figure n4 : Mini laparotomie exposée par l'écarteur d'Alexis, qui a permis le regroupement des lithiases vers l'iléon et leur extraction par une entérotomie

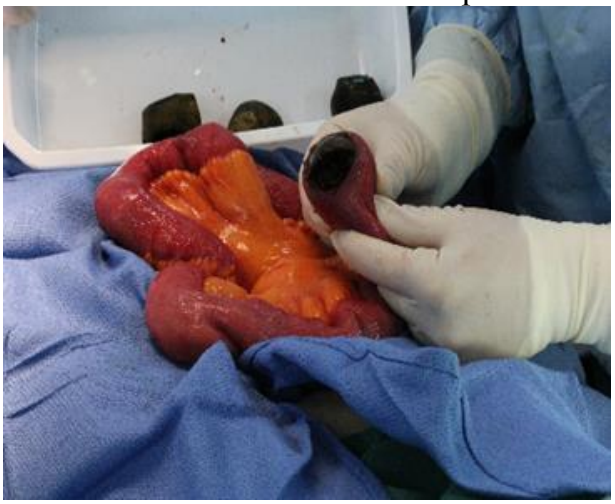

Figure n5: Entérolithotomie sur la face anti mésentérique iléale

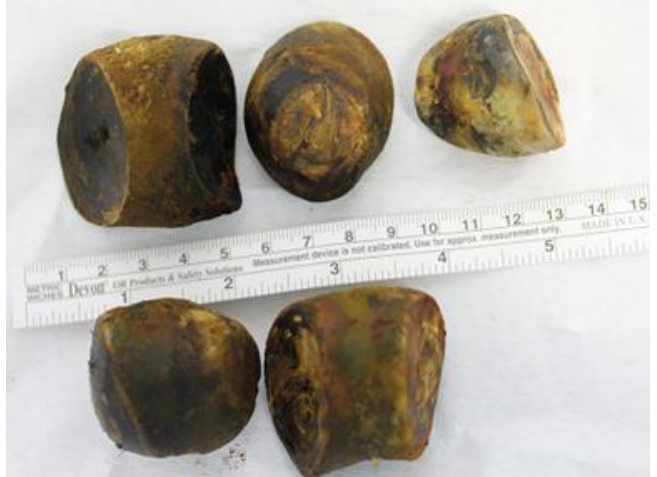

Figure n6: cinq lithiases extraites

Les suites opératoires ont été simples, avec une reprise normale du transit au troisième jour. Des complications post opératoires minimes ont été notés : une rétention aiguë d'urine à $\mathrm{J} 1$ après l'ablation de la sonde vésicale (sur terrain d'hypertrophie bénigne de la prostate) et une infection urinaire à Escherichia Coli et Candida Albicans. Il a été traité médicalement avec une antibiothérapie (Quinolone) et un traitement antifongique.

\section{Discussion}

L'iléus biliaire est une pathologie beaucoup plus fréquente chez la femme âgée, l'âge moyen est de 65 ans (Reisner, 1994) (Rodriguez, 1997). Il 
est causé par le passage d'une macro lithiase entre la vésicule biliaire et la partie du tube digestif au contact. Il se crée l'érosion puis la nécrose et la fistule par l'hyper pression de cette macro lithiase sur le tube digestif. Pour causer une occlusion mécanique, le calcul issu de la vésicule biliaire doit avoir un diamètre $>2$ à 2,5cm (Xin, 2013). Comme l'avaient décrit plusieurs auteurs, l'impaction du calcul peut se faire sur plusieurs parties du tube digestif dont par ordre de fréquence, dans l'iléon $(60,5 \%)$, le jéjunum $(16,1 \%)$, l'estomac $(14,2 \%)$, le colon $(4,1 \%)$ et le duodénum $(3,5 \%)$ (Reisner, 1994) (Lassandro, 2004). Le calcul peut aussi passer et être évacué spontanément dans 1,3\% des cas (Reisner, 1994).

Les manifestations cliniques de l'iléus biliaires sont le plus souvent atypiques, le patient peut avoir une histoire connue de calcul biliaire, mais, le diagnostic de l'iléus biliaire n'est pas forcément le premier énuméré car étant une pathologie très rare. Les symptômes sont ceux d'une occlusion intestinale. Si sur le plan clinique, le diagnostic n'est pas aisé, actuellement le scanner abdominal injecté au produit de contraste, est l'examen le plus performant pour le diagnostic de l'iléus biliaire (Lassandro, 2004). Il précise la localisation exacte et aberrante des macro lithiases dans le tube digestif, il montre des signes indirectes de fistule bilio digestive à savoir l'aérobilie (Lassandro, 2004), mais aussi directement la fistule bilio digestive, vue dans $11,11 \%$ (Lassandro, 2004). Il montre surtout s'il y'a des signes de souffrances digestives ou des signes de péritonite. Il précise aussi l'état de la vésicule biliaire. Une triade radiologique appelée "Rigler's triad » a été décrite en 1946 (Rigler, 1941) et rapportais les trois signes radiologiques comme étant les signes pathognomoniques d'un iléus biliaire. Il s'agit de l'aérobilie, de l'image radiologique d'une lithiase aberrante intra luminale intestinale et les images d'une occlusion grêlique mécanique. Plus tard, en 1978, Baltazar et Schechter (Baltazar, 1978) décrivaient le signe de l'air dans la vésicule biliaire. Avant l'avènement du scanner et ou de l'IRM, le taux de mortalité était plus élevé 7-30\% (Deskoff, 1955) car le diagnostic se faisait tardivement sur 1'ASP (abdomen sans préparation). Actuellement le diagnostic peut se faire aisément par les moyens radiologiques plus performants dont le scanner abdominal injecté, avec une sensibilité de $93 \%$ (Yu, 2005). Par ailleurs, des études récentes ont démontré que ce taux de mortalité et ou de morbidité peut être amélioré avec la méthode de prise en charge la moins invasive possible. Il n y'a pas de procédure uniforme à ce jour, et la méthode de prise en charge prête beaucoup à discussion. Plusieurs questions se posent à savoir, après la levée de l'occlusion, faut-il fermer la fistule biliaire ? Faut-il réaliser la cholécystectomie d'emblée ? Plusieurs méthodes ont été décrites dans la littérature et chacune rapportées avec ses inconvénients et ses avantages: L'enterolithotomie seule, la résection chirurgicale intestinale seule, la résection intestinale associée à la fermeture 
de la fistule. Enfin il a été décrit de traiter tout en même temps, c'est à dire de lever l'occlusion mais aussi de réaliser la fermeture de la fistule et une cholécystectomie d'emblée appelé le «one stage procedure».

Cette technique a été décrite afin d'éviter la récidive et aussi le cancer de la vésicule biliaire selon certains auteurs (Reisner, 1994). En 1929, Holz (Yu, 2005), Fraser en 1954 (Warshaw, 1966) et plus tard Welch en 1957 (Welch, 1957) avaient décrit cette technique comme étant la meilleure, elle permet d'éviter la récidive de la maladie et surtout elle prévient le cancer de la vésicule biliaire dans $15 \%$ des cas (Clavien, 1990).

D'autre part, l'enterolithotomie réalisée seule est rapportée pour certains comme étant la meilleure méthode car ayant le moins de complications post opératoires (Halabi, 2014). Cette méthode reste la moins invasive possible et permet une fermeture spontanée de la fistule dans plus de 50\% des cas (Clavien, 1990). On estime que l'état local d'inflammation ne permet pas la fermeture de la fistule et de la cholécystectomie dans des conditions optimales, au risque de plaie biliaire et ou vasculaire très élevé. Par contre, elle est réservée chez des patients très bien sélectionnés. C'est là où le rôle du scanner abdominal injecté entre en jeu, en permettant d'éliminer les signes de complications ou pas. Mieux, d'autres auteurs pensent que lorsqu'il y a un doute sur des lithiases vésiculaires résiduelles, l'échographie per opératoire est essentielle (Doogue, 1998).

Dans notre cas, une entérolithotomie seule avait été faite, l'étage sus méso colique n'avait pas été manipulé.

Aujourd'hui la laparoscopie est de plus en plus utilisée dans la prise en charge de l'iléus biliaire, elle atteint $10 \%$. Le but de cet abord, est d'être le moins invasif possible afin d'éviter des complications chez des patients déjà grabataires. (Shiwani, 2010) (Soto, 2001). La laparoscopie assistée, rapportée par Sarli et al (Sarli, 1998) consiste à regrouper les lithiases à un même endroit, au niveau de l'iléon, idéal pour une mini laparotomie, lorsqu' il devient plus compliqué de continuer en laparoscopie. Le rôle de la laparoscopie n'est pas clairement défini, elle est utilisée le plus souvent que lorsqu'il s'agit d'une enterolithotomie seule. On note un trop fort taux de conversion lorsque la fermeture de la fistule est envisagée. Il est retrouvé un taux de conversion de 11\% dans la série de Halabi (Halabi, 2014) (Moberg, 2007). En cas de syndrome de Bouveret (lithiase impactée dans le duodénum), le traitement endoscopique par la fibroscopie œsogastro duodénale est de plus en plus utilisé en première intention, (Arif, 2013) (Arnd, 2010). Mais le plus souvent la grande taille de la lithiase reste l'élément responsable de l'échec de ce traitement. D'autres techniques modernes comme la lithotripsie au laser (Maiss, 2004) ou la lithotripsie extra corporéale (Sackmann, 1991) ont aussi été utilisées, toute fois le risque de 
ces méthodes reste l'iléus biliaire distal sur des fragments de lithiases (Moberg, 2007) (Englert, 2012).

\section{Conclusion}

L'iléus biliaire est une pathologie rare, responsable d'occlusion intestinale. Le diagnostic doit être évoqué chez des patients âges ayant une histoire de lithiase vésiculaire connu. Actuellement le scanner abdominal injecté reste le meilleur examen de référence, permettant un diagnostic rapide et précis. La prise en charge doit être urgente et la moins invasive possible. L'enterolithotomie seule est la méthode la plus utilisée, mais aussi le « one stage procédure » chez les patients bien sélectionnés.

\section{References:}

1. Deskoff SL. Et al; Gallstone ileus a report of 12 cases. Ann Surg 1955;142: 52-65

2. Halabi WJ, Celeste Y. Kang, NoorKetana, Kelly J.Lafaro, Vinh Q.Nguyen, Michael J.Stamos, David K.Imagawa, and Aram N.Demirjian : Surgery of gallstoneileus;Ann of Surg, vol259, Num2, Fev 2014

3. Reisner RM, Cohen JR, gallstone ileus a review of 1001 reported cases; Ann of Surg1994; 60:441-446

4. Rodriguez JC, Casado F, Fernandez MJ, Morales DJ, Naranjo A, cholecystectomy and fistula closure vsenterolithomy alone in GI Br J Surg, 1997;84:634-637

5. Xin -Zheng D, Guo-Qiang L, Feng Z, Xue-Hao W, Chuan-Yong Z, Gallstone iléus: case report and littérature review world j gastroenterrol. 2013 sept 7; 19 (33) :5586-5589

6. Lassandro F, Gagliardi N, Scuderi M, Pinto A, Gatta G, Mazzeo R, Gallstone iléus analysis of radiological findings in 27 cases; Euro J Radiol 2004;50:23-29

7. Rigler LI, Borman CN, Noble JF, .Gallstone obstruction. Pathogenesis and roentgen manifestations. JAMA 1941;117:1753-9

8. Baltazar EJ, Schechter LS. Air in gallbladder: afrequent finding in gallstone ileus. AJR Am J Roentgenol 1978; 131:219-22

9. Yu CY, Lin CC, Shyu RY, Hsieh CB, Wu HS, Tyan YS, et al, value of CT in the diagnosis and management of gallstone ileu. Word $\mathrm{J}$ Gastro 2005; 11:2142-2147

10. Warshaw AL, Bartlett MK, choice of operation of gallstone intestinal obstruction. Ann Surg 1966;164:1051-5

11. Fraser WJ intestinal obstruction by gallstone. Br J Surg 1954;42:210

12. Welch JS, Huizenga KA, Robert SE. Recurrent intestinal obstruction due to gallstone. Proc Staff Meet Mayo Clinic 1957; 32:628. 
13. ClavienPA, Richon J, Burgan S et al. A gallstoneileus. Br J Surg 1990;77:737-42

14. Doogue MP, Choong CK, Frizelle FA. Recurrent gallstone ileus, underestimated. Aus N Z J Surg 1998; 68:755-756

15. Shiwani MH, Ullah Q, Laparoscopic enterolithotomy is a valid option to treat gallstone iléus. JSLS $2010 ; 14: 282-285$.

16. Soto DJ, Evan SJ,Kavic MS. Laparoscopic management of gallstone iléus. JSLS $2001 ; 5: 279-285$.

17. Sarli L, Pietra N, Costi R, GobbiS.Gallstone iléus : laparoscopicassistedenterolithotomy. J Am CollSurg 1998 ; 186 :371-371.

18. Moberg AC, Montgomery A. Laparoscopically assisted or open enterolithotomy for gallstone iléus. Br J Surg. 2007 ; 94 :53-57.

19. Arif Z, Gemma I, Jainudeen KA. Bouveret syndrome presenting with acute pancreatitis a very rare and challenging vari nt of gallstone iléus. International Journal of Surgery Case report 4 (2013) 528-530.

20. Arnd G, Jürgen Z, Guido W, Bernhard F. Development of a duodénal gallstone iléus with gastric outlet obstruction (Bouveret syndrome) four months gallstone disease with cholecystitis and cholangitis: a case report. Giese et al Journal of Medical Case reports 2010, $4 ; 376$.

21. Maiss J, Hochberger J, Hahn EG, Lederer R, and Schneider HT, Muehldorfer S. Successful laserlithotripsy in Bouveret'S syndrome using a new frequency doubled doublepulseNd: Yag laser (Freddy). Scan J Gastroenterol 2004 ; 39 : 791-794.

22. Sackmann M, Holl J, Haerlin M, and al. Gallstone iléus successfully treated by shock-wave lithotripsy. Dig Dis Sci 1991 ; 36 : 1794-1795.

23. Englert ZP, Love K, Marilley MD, Bower CE. Bouveretsyndrome: gallstone iléus of the duodénum. SurgLaparoscEndoscPercutan Tech $2012 ; 22: 301-3$. 